\title{
Comparison of Compliance Test for Soil of Ministry of Environment with an in vitro Soil Oral Bioaccessibility Test for Lead and Cadmium
}

\author{
Nyein Nyein AUNG and Jun YOSHINAGA \\ Institute of Environmental Studies, University of Tokyo \\ (7-3-1 Hongo, Bunkyo, Tokyo 113-0033)
}

[Received January 13, 2004]

\begin{abstract}
Summary
The compliance test for the evaluation of the health risk arising from soil ingestion of contaminated sites employed in the new Contamination Control Law by Ministry of Environment, Government of Japan (MoE method), was evaluated against a gastric-condition simulation soil oral bioaccessibility test, established by Solubility/Bioaccessibility Research Consortium, USA (SBRC method). The extracted elemental concentrations, viz., cadmium and lead, of soil certified reference materials (CRMs) and soil samples obtained by applying both tests were determined and compared. The MoE method extracted more lead from soil than did the SBRC method, while the extracted cadmium by the MoE method was found to be less than the oral bioavailibility. The importance of other factor(s) than acidity of extractant was indicated in the cadmium results. Further characterization was warranted for the MoE method as a means to evaluate the human health risk of a contaminated site.
\end{abstract}

Key words: soil, lead, cadmium, compliance test of Ministry of Environment, bioaccessibility

\section{Introduction}

During the past decade, it has been recognized that health risk assessment of contaminated sites should be based on the contaminant level in soil absorbable to humans; not on the total concentration in the soil ${ }^{1}$. This is because not all of the fractions of the contaminants present in soil are reachable to the blood circulation system of human beings. Here, the fraction that is absorbable in the gastrointestinal (GI) tract is defined as bioaccessible fraction and that reachable to blood stream is defined as bioavailable fraction ${ }^{2}$.

The oral bioavailability of soil contaminants was usually estimated by in vivo methods, in which test animals are fed with contaminants in a soil matrix. Due to the high expenses and time-constraints of in vivo methods, a variety of in vitro bioaccessibility testing methods, which simulate the human physiological conditions of GI tract, have been developing nowadays for estimating the oral bioavailability of the soil contaminants ${ }^{1-3}$. The reliability of such in vitro tests should ideally be validated by in vivo tests; otherwise the method may not be of relevance to the measures of human health effects.

The Ministry of Environment, Government of Japan, proclaimed the new Soil Contamination Control Law (Law No. 53 of 2002) on May 29, 2002, and became effective on February 15, 2003". One of the compliance tests in this Law (Notification 19) applies 1M $\mathrm{HCl}$ to simulate the stomach condition of human being. In this short report, we compared the compliance test of Ministry of Environment Notification 19 (MoE method) with an in vitro soil oral bioaccessibility test developed by Solubility/Bioavailability Research Consor- 
tium (SBRC method) of the United States by using soil certified reference materials (CRMs) and some real soil samples. This SBRC method, which simulates only the stomach condition of humans, is currently adopted by British Geological Survey and nominated as one of the candidate methods for standardizing bioaccessibility test at the European platform on bioavailability and bioaccessibility, the BioAvailability Research Group Europe (BARGE) $)^{5}$. In addition, this SBRC test was validated through the swine in vivo tests ${ }^{1,2}$. This indicates that the leached concentration of elements under the test conditions is predictive of oral bioavailability in the animal model and thus the comparison of the results by MoE and SBRC methods may allow us to indirectly elucidate the $\mathrm{MoE}$ method in terms of bioavailability measures.

\section{Materials and Method}

\section{Samples}

Soil compliance test of Ministry of Environment Notification 19 (MoE method) and the gastric condition simulation in vitro bioaccessibility test (SBRC method) were performed for three soil CRMs [SRM 2711 Montana soil from the National Institute of Standards and Technology (NIST), USA, JSAC 0401 from Japan Society for Analytical Chemistry (JSAC), and JSO-1 from Geological Survey of Japan], two industrially polluted soil samples No. 2 and No. $5^{6)}$ and two playground surface soil samples. NIST SRM 2711 was included for validation of our SBRC operation because of the availability of the data by SBRC method. The JSAC 0401 and JSO-1 are CRMs of Japanese soil origin. Two playground surface soil samples were collected from the play areas of public parks in Tokyo in June 2002 and their lead content has been reported elsewhere ${ }^{7}$. In order to evaluate the precision of soil analysis, more than two independent sets of extraction-measurement were made for the samples except where sample amount was limited.

\section{SBRC Method ${ }^{1,5)}$}

The SBRC method is recommended typically for lead bioaccessibility measures. In this method, the element concentration extracted into the simulated gastric juice out of total content is measured and that fraction is defined as bioaccessiblity in that particular soil. The bioaccessibility in this report is calculated by the following equation:
Bioaccessibility $(\%)=[$ Contaminant mobilized from soil during extraction $(\mu \mathrm{g})$ ] / [Total contaminant present in soil $(\mu \mathrm{g})] \times 100$

One gram of dry soil was added to $100 \mathrm{~m} \ell 0.4 \mathrm{M}$ glycine solution, which had been adjusted to $\mathrm{pH} 1.5$ with $\mathrm{HCl}$. The mixture was end-over-end rotated at $37{ }^{\circ} \mathrm{C}$ at $30 \mathrm{rpm}$ for $1 \mathrm{hr}$. Sample was transferred into a disposable polypropylene (PP) syringe and filtered through $0.45 \mu \mathrm{m}$ cellulose acetate disk filter (Sartrius, Germany). The $\mathrm{pH}$ of the filtrate was monitored not to exceed $\pm 0.5 \mathrm{pH}$ unit of the starting $\mathrm{pH}$. Analysis was performed within one week after in vitro test by using inductively coupled plasma mass spectrometer (ICP-MS, HP 4500, Yokogawa Analytical Systems Inc., Japan) with internal standardization (yttrium for arsenic and cadmium and bismuth for lead).

Quality control of our operation of the SBRC method was conducted by comparing both the extracted concentration and the extracted fraction (bioaccessibility) obtained in this research and those obtained by the other researchers.

\section{MoE method}

We strictly followed the procedure specified in the MoE method ${ }^{4}$ : $6.00 \mathrm{~g}$ soil/CRM was put into a $500 \mathrm{~m} \ell$ PP bottle. Two hundred milliliter of $1 \mathrm{M} \mathrm{HCl}$ was added to the bottle and the bottle was horizontally shaken for 2 hrs on a shaker (TAITEC, Japan) at shaking width of $4 \sim 5 \mathrm{~cm}$ and 200 shaking/min at room temperature. Upon completion, the bottle was let stand for 10 30 min., which was followed by filtration through a $0.45 \mu \mathrm{m}$ cellulose acetate disk filter and measurement by ICPMS with internal standards. Moisture content of the sample was measured as the weight loss of soil sample after heating at $110{ }^{\circ} \mathrm{C}$ in an electric oven for $4 \mathrm{hrs}$ and it was used for correcting the element concentration in soil samples. For quality control of the $\mathrm{MoE}$ method, the concentrations of the CRM JSAC 0401 of this study were compared with those obtained from JSAC.

For both of the methods, appropriate procedural blanks were prepared and concurrently analyzed to find the negligible contribution to the results.

\section{Chemicals}

The reagents used throughout the experiments were of analytical grade or higher purity and they 
were purchased from Kanto Chemicals Co., Japan. An ICP multi-element standard solution from EM Science (NJ, USA) was used for the calibration of ICPMS. The working standard solutions were prepared fresh daily by serial dilutions with nitric acid and Millipore-purified water.

\section{Results and Discussion}

Table 1 shows the comparison of elemental concentrations in the extract of the SBRC method in this study and those reported in the previous studies. As shown in this table, the reproducibility of our results for arsenic, cadmium and lead was $<4 \%$ as expressed as relative standard deviation (RSD) and the average values of both extracted concentration $(\mathrm{mg} / \ell)$ and extracted fraction (\%) obtained in this research agreed well with those of other researchers ${ }^{1,5}$. This indicated the validity of our SBRC operation and ICPMS measurement. A slightly lower extracted fraction was, however, observed for cadmium than that reported by Oomen et al.5. This can be due to the fact that they divided (above-mentioned equation of $\%$ bioaccessibility) the elemental concentrations in the extract by the average total element contents determined by a number of laboratories (numbers unidentified), which were at the lower margin of the certified values, whereas the certified values were used in calculating our results.

Table 2 illustrates the comparison of the elemental concentrations in JSAC 0401 by the MoE method in this study and those obtained from $\mathrm{JSAC}^{8}$. We intended to compare the elemental concentration of arsenic, cadmium and lead. However, determination of arsenic concentration in $1 \mathrm{M} \mathrm{HCl}$ extractant by ICPMS was not appropriate because of $\mathrm{ArCl}$ interference and thus we excluded arsenic data by the MoE method hereafter. It has to be stressed, however, that arsenic was accurately determinable in the extractant obtained by the SBRC method as demonstrated in Table 1. The concentrations of lead and cadmium were consistent with JSAC compiled values indicating our MoE operation was also valid as was the SBRC operation for these two elements (Table 1).

Table 1 Comparison of the element concentrations in the extract of NIST 2711 CRM obtained by the SBRC method

\begin{tabular}{|c|c|c|c|}
\hline & This study & Other & Source \\
\hline Extracted As & $0.52 \pm 0.01 \mathrm{mg} / \ell^{\mathrm{a}}$ & $0.59 \pm 0.09 \mathrm{mg} / \ell^{\mathrm{c}}$ & Kelley et al. ${ }^{1)}$ \\
\hline Extracted fraction (As) & $(50 \pm 1) \%^{d}$ & $(59 \pm 2) \%^{\mathrm{e}}$ & Oomen et al. ${ }^{5)}$ \\
\hline Extracted Cd & $0.358 \pm 0.006 \mathrm{mg} / \ell^{\mathrm{a}}$ & Not available & \\
\hline Extracted fraction $(\mathrm{Cd})$ & $(88 \pm 2) \%^{\mathrm{d}}$ & $(99 \pm 4) \%^{\mathrm{e}}$ & Oomen et al. ${ }^{5)}$ \\
\hline Extracted $\mathrm{Pb}$ & $10.1 \pm 0.4 \mathrm{mg} / \ell^{\mathrm{b}}$ & $9.22 \pm 1.50 \mathrm{mg} / \ell^{\mathrm{c}}$ & Kelley et al. ${ }^{1)}$ \\
\hline Extracted fraction $(\mathrm{Pb})$ & $(88 \pm 3) \%^{f}$ & $(90 \pm 2) \%^{e}$ & Oomen et al. ${ }^{5)}$ \\
\hline \multicolumn{4}{|c|}{${ }^{a}$ Mean of duplicated measurements and standard deviation } \\
\hline \multicolumn{4}{|c|}{${ }^{\mathrm{b}}$ Mean of 5 measurements and standard deviation } \\
\hline \multicolumn{4}{|c|}{${ }^{\mathrm{c}}$ Mean of 18 blind splits measurements in 4 independent laboratories } \\
\hline \multicolumn{4}{|c|}{$\begin{array}{l}{ }^{d} \text { Mean of calculated results based on certified value and extracted values of } 2 \\
\text { measurements }\end{array}$} \\
\hline \multicolumn{4}{|c|}{${ }^{\mathrm{e}}$ Mean of reported values by a number of laboratories } \\
\hline
\end{tabular}


Table 2 Element concentrations in JSAC 0401 obtained by MoE method

\begin{tabular}{ccc}
\hline & $\mathrm{Cd}(\mathrm{mg} / \mathrm{kg})$ & $\mathrm{Pb}(\mathrm{mg} / \mathrm{kg})$ \\
\hline This study $^{\mathrm{a}}$ & $3.40 \pm 0.51$ & $14.0 \pm 0.4$ \\
JSAC $^{\mathrm{b}}$ & $3.75 \pm 0.27$ & $13.8 \pm 1.0$ \\
\hline${ }^{\mathrm{a}}$ Mean of triplicated samples and standard deviation \\
${ }^{\mathrm{b}}$ Information values; mean values reported from & 12 collaborating \\
\multicolumn{2}{l}{ laboratories and standard deviation } & ${ }^{8)}$
\end{tabular}

The extracted lead fraction by the $\mathrm{MoE}$ method was as large as $>85 \%$ except for one sample (54\%, JSAC0401 CRM) indicating the efficient extracting characteristics of the MoE method. Moreover the MoE method extracted more lead from soil than did the SBRC for all of the seven samples examined in this study (Table 3). This should be due to stronger acidity of the extractant used in the $\mathrm{MoE}$ method $(1 \mathrm{M} \mathrm{HCl})$ than that in the SBRC (pH 1.5 glycine solution) and this was in agreement with our expectation. This result indicates that the MoE method extracts more lead than the bioavailable fraction in soil allowing risk characterization of soil pollution from safer side.

However, this did not hold true to cadmium: the SBRC extracted more cadmium from some samples than did the MoE (Table 3). It can be assumed that other factor(s) than acidity of extractant affected the extractability of cadmium. It is widely recognized that leaching of metals from solid sample is a considerably complex process in which a number of factors, such as extractant composition, liquid-solid (L/S) ratio, duration of extraction etc., are involved. Particularly $\mathrm{pH}$ of the extractant is a key factor responsible to the metal leaching from solid samples ${ }^{5,9}$. In the present $\mathrm{MoE}^{-}$ SBRC comparison, key differences included extractant composition (1M HCl vs $0.4 \mathrm{M}$ glycine of $\mathrm{pH} 1.5$ ), L/S ratio (3:100 vs 1:100), duration of extraction (2 hrs vs $1 \mathrm{hr}$ ), and extraction temperature (room temperature vs $37{ }^{\circ} \mathrm{C}$ ). Among these, the extractant composition was the most likely factor to affect cadmium extractability from soil matrix. Although the acidity applied in SBRC method was weaker than the $1 \mathrm{M} \mathrm{HCl}$ used in the MoE method, the complexing capacity of glycine may have something to do with cadmium ex- tractability from soil and/or solubility or stability of cadmium in the solution. Higher extraction rate of cadmium in the presence of organic matter in the extractant was shown in Oomen et al $(2002)^{5)}$; bioaccessibility of cadmium in a soil sample increased from 38 $\%$ to $62 \%$ under the presence of milk powder in the extractant, while it was 31 to $40 \%$ and 30 to $44 \%$ for lead and arsenic, respectively. This tendency was also true to the other two soil samples tested. This result indicated that higher mobilization of metals in soil into extractant by complex formation was more pronounced in the case of cadmium than for other metals. The MoE method, thus, may not necessarily allow stricter risk characterization for cadmium in soil as it does for lead in spite of the stronger acidity of the extractant than that of stomach condition. Since the number of samples is limited in this short report, further study was warranted to fully characterize the extraction of other contaminants from a variety of soil matrices by the MoE method. Comparison with an established extracting method that can be representative of bioavailability, such as SBRC method, will be an effective means to this end.

It should also be noted that the present results for soil CRMs (NIST SRM 2711, JSAC 0401 and JSO1 ), all three are commercially available, by the $\mathrm{MoE}$ method will be valuable as a crosscheck data for the laboratories that are involved in soil analysis since there are no soil CRMs with information on the concentrations of elements extractable by $1 \mathrm{M} \mathrm{HCl}$ is available at present.

\section{ACKNOWLEDGEMENT}

The authors appreciate Dr. T. Toyoguchi, Environ- 
Table 3 Comparison of soil contaminants extracted by the MoE method and by the SBRC method for the certified reference materials and other soil samples samples

\begin{tabular}{l|ccc|ccc}
\hline \multirow{2}{*}{ samples } & \multicolumn{3}{|c|}{$\mathrm{Cd}(\mathrm{mg} / \mathrm{kg})$} & \multicolumn{3}{c}{$\mathrm{Pb}(\mathrm{mg} / \mathrm{kg})$} \\
\cline { 2 - 7 } & Total & $\mathrm{MoE}$ & $\mathrm{SBRC}$ & total & $\mathrm{MoE}$ & SBRC \\
\hline NIST 2711 & $41.70 \pm 0.25^{\mathrm{a}}$ & $29.6 \pm 0.1^{\mathrm{b}}$ & $36.2 \pm 0.9^{\mathrm{b}}$ & $1162 \pm 32^{\mathrm{a}}$ & $1020 \pm 20^{\mathrm{b}}$ & $994 \pm 43^{\mathrm{c}}$ \\
JSAC 0401 & $4.25 \pm 0.41^{\mathrm{a}}$ & $3.40 \pm 0.51^{\mathrm{b}}$ & $3.04 \pm 0.09^{\mathrm{b}}$ & $26 \pm 4^{\mathrm{a}}$ & $14.0 \pm 0.4^{\mathrm{b}}$ & $11.6 \pm 0.2^{\mathrm{b}}$ \\
JSO-1 & $\mathrm{NA}$ & 0.0331 & 0.0806 & & & \\
& & $\pm 0.0036^{\mathrm{b}}$ & $\pm 0.0043^{\mathrm{b}}$ & $12^{\mathrm{a}}$ & $11.5 \pm 1.2^{\mathrm{b}}$ & $4.78 \pm 0.20^{\mathrm{b}}$ \\
No.2 & $\mathrm{NA}$ & $219^{\mathrm{d}}$ & 161,143 & $\mathrm{NA}$ & $10,600^{\mathrm{d}}$ & 7140,6120 \\
No.5 & $\mathrm{NA}$ & $\mathrm{NA}$ & $0.555,0.112$ & $\mathrm{NA}$ & $585^{\mathrm{d}}$ & 437,347 \\
P1 & $0.585^{\mathrm{e}}$ & $0.273,0.240$ & $0.454,0.350$ & $111^{\mathrm{e}}$ & $99.3,88.8$ & $66.1,65.2$ \\
P2 & $0.710^{\mathrm{e}}$ & 0.299 & 0.541 & $114^{\mathrm{e}}$ & 104 & 99.4 \\
\hline
\end{tabular}

${ }^{\text {a }}$ Certified/reference value

${ }^{b}$ Mean of triplicated samples and its standard deviation

${ }^{c}$ Mean of 5 measurements and its standard deviation

$\left.{ }^{\mathrm{d}} \operatorname{Ref} 6\right)$

${ }^{\mathrm{e}}$ Concentration measured by the authors

mental Control Center Co. Ltd., for supplying industrially polluted soil samples. Part of this study was supported by Nissei Foundation.

\section{REFERENCES}

1) Kelley, M.E., Brauning, S.E., Schoof, R.A. and Ruby, M.V.: Assessing oral bioavailability of metals in soil. Battelle Press. Ohio (2002)

2) Ruby, M.V., Schoof, R., Brattin, W., Goldade, M., Post, G., Harnois, M., Mosby, D.E., Casteel, S.W., Berti, W., Carpenter, M., Edwards, D., Cragin, D. and Chappel, W.: Advances in evaluating the oral bioavailoability of inorganics in soil for use in human health risk assessment. Environ. Sci. Technol., 33, 3697-3705 (1999)

3) Yamada , A., Ono, Y., Kida, A. and Namiki, K.: Assessment with in vitro screening test for bioavailability of heavy metals in road sediment. kankyokagaku, 15 (6), 407-414 (2002) (in Japanese with English abstract)
4) Home page of Ministry of Environment, Government of Japan

http://www.env.go.jp/water/dojo/law/kokuji.html

5) Oomen, A.G., Hack, A., Minekus, M., Zeijdner, E., Cornelis, C., Verstraete, W., Wiele, T.V.D., Wragg, J., Rompelberg, C.J.M., Sips, A.J.A. and Wijnen, J.H.V.: Comparison of five in vitro digestion models to study the bioaccessibility of soil contaminants. Environ. Sci. Technol., 36, 3326-3334 (2002)

6) Suzuki, S., Kida, A., Sakai, S. and Morita, M.: Evaluation of an interlaboratory comparison for inorganic compounds in soils. Haikibutsu Gakkai Shi, 14, 105-113 (2003) (in Japanese)

7) Aung, N.N., Yoshinaga, J. and Tanaka, A.: Lead in the Playground Soil: Significance in Exposure to Children and its Contamination Source (submitted)

8) Personal communication from JSAC (2004)

9) Sakai, S., Mizutani, S. and Takatsuki, H.: Leaching tests for waste materials. Haikibutsu Gakkai Shi 7, 383-393 (1996) (in Japanese) 